Pacific Journal of Mathematics

RATIONAL STRUCTURES ON 3-MANIFOLDS

aN AKBULUT AND HENRY CHURCH UL KING 


\section{RATIONAL STRUCTURES ON 3-MANIFOLDS}

\section{Selman Akbulut and Henry King}

In this paper we prove a conjecture of Nash for 3-manifolds. That is, we show that any two 3-manifolds are rationally equivalent. This means that by operations of topologically blowing up along smooth submanifolds (i.e. points and curves in this case) we can make any two 3-manifolds diffeomorphic to each other.

In [Na] Nash conjectured that any two, closed, smooth connected manifolds of the same dimension can be made diffeomorphic after topologically blowing them up along submanifolds. ${ }^{1}$ In [T] a counterexample to this conjecture was announced, but the proof is unfortunately not correct. This conjecture is clearly true for 2-manifolds, since blowing up a surface at a point has the affect of connected summing with an $\mathbb{R} \mathbb{P}^{2}$. In the case of 3-manifolds we had reduced this conjecture to a problem about ' $\mathbb{Z}_{2}$-framed link calculus" in $S^{3}$ as reported in [A1]. This problem recently has been solved by Nakanishi [N]. Here we present the proofs of these results by showing how [N] along with our results which were announced in [A1] proves the Nash conjecture for 3-manifolds. We would like to thank R. Benedetti for bringing $[\mathbf{N}]$ to our attention. We first need to recall the definition of topological blow-up:

1. Blowing up. Let $N \subset M$ be closed smooth manifolds with $k=$ $\operatorname{dim}(M)-\operatorname{dim}(N)$. Let $\pi: E \rightarrow N$ be the normal $\mathbb{R}^{k}$-bundle, and $\hat{\pi}: \widehat{E} \rightarrow N$ be the associated $O(k)$-bundle. $O(k)$ acts on $\mathbb{R} \mathbb{P}^{k}-$ $[1: 0: \cdots: 0]$ as follows: If $A \in O(k)$, and $\left[x_{0}: \cdots: x_{k}\right] \in \mathbb{R} \mathbb{P}^{k}-$ $[1: \cdots: 0]$, then $A\left[x_{0}: \cdots: x_{k}\right]=\left[x_{0}: y_{1}: \cdots: y_{k}\right]$, where $\left(y_{1}, \ldots\right.$, $\left.y_{k}\right)=A\left(x_{1}, \ldots, x_{k}\right)$. In particular $O(k)$ acts on $\mathbb{R}^{k-1}$. Let

$$
\mathbb{R} \mathbb{P}^{k}-[1: 0 \cdots: 0] \rightarrow E^{\prime} \stackrel{\pi^{\prime}}{\rightarrow} N \quad \text { and } \quad \mathbb{R} \mathbb{P}^{k-1} \rightarrow E^{\prime \prime} \stackrel{\pi^{\prime \prime}}{\rightarrow} N
$$

be the associated bundles, i.e.

$$
E^{\prime}=\widehat{E} \times_{O(k)}\left(\mathbb{R} \mathbb{P}^{k}-[1: \cdots: 0]\right) \quad \text { and } \quad E^{\prime \prime}=\widehat{E} \times_{O(k)} \mathbb{R} \mathbb{P}^{k-1} .
$$

\footnotetext{
hold.

${ }^{1}$ Here we state the topological version of his conjecture since the algebraic version does not
} 
We have the natural inclusions $E^{\prime \prime} \subset E^{\prime}$. The map:

$$
\begin{aligned}
& \tau: \mathbb{R} \mathbb{P}^{k}-[1: 0: \cdots: 0] \rightarrow \mathbb{R}^{k}-0 \text { defined by } \\
& \tau\left[x_{0}: \cdots: x_{k}\right]=\frac{x_{0}}{\sum x_{i}^{2}}\left(x_{1}, \ldots, x_{k}\right)
\end{aligned}
$$

commutes with the $O(k)$-action and hence induces a diffeomorphism $\alpha$ :

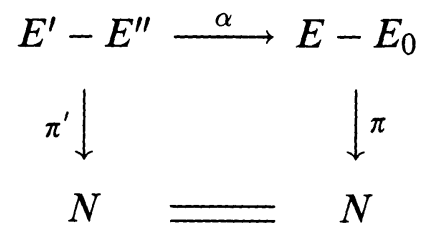

where $E_{0}=0$-section of $E=N$.

Definition. The blow up of $M$ along $N$ is the smooth manifold obtained by glueing $M-N$ and $E^{\prime}$ with the map $\alpha$ (after identifying $E$ with a tubular neighborhood of $N$ ), and it is denoted by

$$
B(M, N)=(M-N) \cup_{\alpha} E^{\prime} .
$$

There is the natural projection map: $\pi: B(M, N) \rightarrow M$ given by:

$$
\left.\pi\right|_{M-N}=\text { inclusion, and }\left.\pi\right|_{E^{\prime \prime}}=\pi^{\prime \prime} .
$$

Hence $\tau$ is a diffeomorphism over $M-N$, and is the bundle map $\pi^{\prime \prime}$ over $N$.

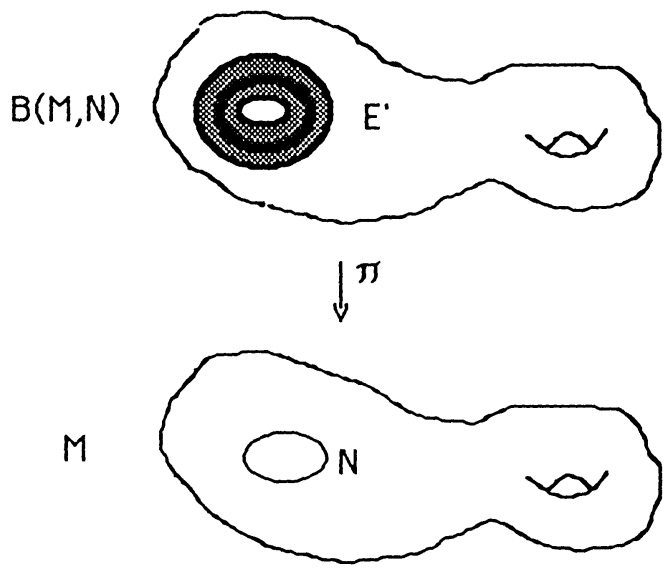

FIGURE 1 
In particular if $N \subset M$ has a trivial normal bundle then

$$
B(M, N)=\left(M-N \times \operatorname{int}\left(B^{k}\right)\right) \cup_{\partial}\left(N \times \mathbb{R} \mathbb{P}_{0}^{k}\right)
$$

where $\mathbb{R} \mathbb{P}_{0}^{k}=\mathbb{R} \mathbb{P}^{k}-\operatorname{int}\left(B^{k}\right)$ is the punctured projective space. For example, if $p \in M$, then $B(M, p)=M \# \mathbb{R} \mathbb{P}^{k}$, where $k=\operatorname{dim}(M)$, and more generally $B(M \times Q, p \times Q)=\left(M \# \mathbb{R} \mathbb{P}^{k}\right) \times Q$, where $Q$ is any smooth manifold. When $N$ and $M$ are nonsingular algebraic sets then $B(M, N)$ has a natural algebraic structure.

Definition. We say two closed manifolds $M^{m}$ and $Q^{m}$ are birationally equivalent, and denote it by $M \sim Q$, if there are sequences of blow ups along closed submanifolds:

$$
\begin{gathered}
M \leftarrow M_{1} \leftarrow M_{2} \leftarrow \cdots \leftarrow M_{k}, \\
Q \leftarrow Q_{1} \leftarrow Q_{2} \leftarrow \cdots \leftarrow Q_{r}
\end{gathered}
$$

so that $M_{k} \approx Q_{r}$, i.e., they are diffeomorphic.

Birational equivalence is in fact an equivalence relation (a fact which is not used in this paper). This is because any two different blowups of $M$ admit a common blowup. We are now ready to state the conjecture of Nash [Na] in our context:

Conjecture. Any two closed smooth manifolds of the same dimension are birationally equivalent.

To understand this problem we need to see how 3-manifolds transform under blowing up operations:

2. Framed link pictures of blow-up. Recall that any closed 3-manifold $M^{3}$ bounds a 4-manifold $W^{4}$. Furthermore we can take $W$ to be $B^{4}$ with 2-handles attached if $M$ is oriented, and $B^{3} \tilde{\times} S^{1}$ (the twisted $B^{3}$-bundle over $S^{1}$ ) with 2-handles attached if $M$ is unoriented. So, $M$ can be represented by a framed link in either $S^{3}$ or in $S^{2} \tilde{\times} S^{1} . S^{2} \tilde{\times} S^{1}$ is the boundary of $B^{3} \tilde{\times} S^{1}$, which is $B^{4}$ with one handle attached. By using the notation of [A2], $B^{3} \tilde{\times} S^{1}$ can be represented by a pair of 3-balls, which is the attaching set, $S^{0} \times B^{3}$, of the 1-handle $B^{1} \times B^{3}$.
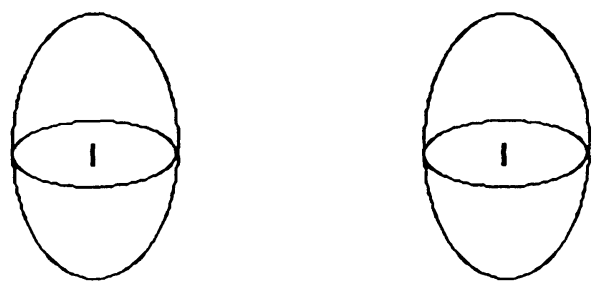

FIGURE 2 


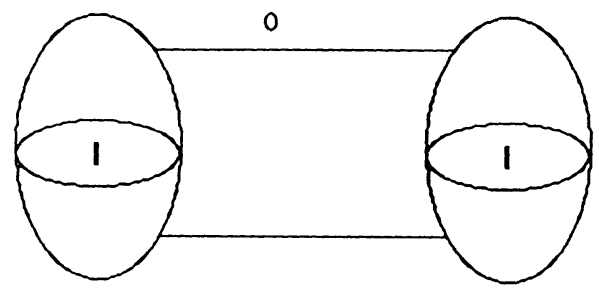

FIGURE 3

If we imagine coordinate axes in the centers of these balls then the 1-handle identifies the boundaries of these two balls by the map $(x, y, z) \mapsto(x,-y,-z)$. So, in general $M$ is represented by a framed link, along with possibly a pair of balls. In case the balls are present (i.e. in $S^{2} \tilde{\times} S^{1}$ ) the link could go over the 1-handle. For example $\mathbb{R} \mathbb{P}^{2} \times S^{1}$ is given by Fig. 3 [A2].

We now want to see how the blowing up operation changes a framed link picture of a 3-manifold $M^{3}$. Since blowing up codimension 1 submanifolds does not change the manifold, we only have to consider blowing up $M^{3}$ along points and knots.

Blowing up a point $p \in M$. Clearly $B(M, p)=M \# \mathbb{R} \mathbb{P}^{3}$. Hence the framed link of $M$ changes by introducing a disjoint unknotted circle with \pm 2 framing.

Blowing up a knot $\gamma \subset M$.

Case 1. $M=S^{2} \times S^{1}, \gamma=p \times S^{1}, p \in S^{2}$.

Fig. 4 is the framed link picture of $M$. Then $B(M, \gamma)=\mathbb{R} \mathbb{P}^{2} \times S^{1}$ is given by Fig. 5. The dotted circle $\delta$ in the figure is not part of the framed link; it is a picture of the image of a parallel copy of the loop $\gamma$ by the diffeomorphism $(\pi \mid)^{-1}: M-\gamma \rightarrow B(M, \gamma)-\pi^{-1}(\gamma)$.

Case 2. $M=S^{3}$, and $\gamma$ is the unknot.

Fig. 6 is the picture of $\gamma \subset M$. Since the 0 -framed handle $\alpha$ is attached to a parallel copy of $\gamma$, by Case 1 Fig. 7 gives $B(M, \gamma)$. Fig. 7 and Fig. 8 both describe $S^{2} \widetilde{\sim} S^{1}$.

Case 3. $M=S^{2} \tilde{\times} S^{1}, \gamma=p \times S^{1}, p \in S^{1}$.

In this case $B(M, \gamma)=\mathbb{R} \mathbb{P}^{2} \times S^{1}$, i.e. it is the twisted $\mathbb{R} \mathbb{P}^{2}$-bundle over $S^{1}$. So it must be the product bundle $\mathbb{R} \mathbb{P}^{2} \times S^{1}$, which is Fig. 10. Fig. 9 gives the picture of $\gamma \subset M$. Again by inspection we see that the dotted circle $\delta$ in Fig. 10 is the image of a parallel copy of $\gamma$ by the diffeomorphism $(\pi \mid)^{-1}: M-\gamma \rightarrow B(M, \gamma)-\pi^{-1}(\gamma)$. By rotating one of the attaching balls of the 1-handle we can make Fig. 10 diffeomorphic to Fig. 11. 
Case 4. $\gamma \subset M$ has a trivial normal bundle.

By adding a cancelling pair of 2-handles as in Fig. 12 we can draw a picture of an arbitrary knot $\gamma$ which looks unknotted. In this picture the box represents the complicated part of the knot $\gamma$. Then by Case 1, Fig. 13 represents $B(M, \gamma)$.

Case 5. $\gamma \subset M$ has a nontrivial normal bundle.

This means that $\gamma$ has to go through an orientation reversing 1handle an odd number of times. By adding a cancelling pair of 2handles as in Fig. 14, and by sliding over them we can assure that $\gamma$ goes over the 1-handle only once. By sliding one of the attaching balls of the 1 -handle along $\gamma$, we can always assume that $\gamma$ is in the standard form as in Fig. 15. Now by Case 3, Fig. 16 gives $B(M, \gamma)$.

3. $\mathbb{Z}_{2}$-framed link calculus. Framed links are useful tools in deciding whether two 3-manifolds are diffeomorphic. The main result of [K] says that any two framed links in $S^{3}$ represent the same 3manifold if and only if one is obtained from the other by a sequence of "handle-sliding" operations, and introducing or erasing an unknotted circle with \pm 1 framing. These are known as "Kirby moves", and the equivalence class of framed links under these moves is called the "Kirby calculus". One can easily extend this calculus to framed links in $S^{2} \tilde{\times} S^{1}$ and get a similar result for nonorientable 3-manifolds. Here we introduce the following equivalence relation to this calculus, which in a way specializes it to $\mathbb{Z}_{2}$.

Definition. We say that any two framed links are $\mathbb{Z}_{2}$-equivalent, if one can be obtained from the other by a sequence of Kirby moves, twisting across any strand \pm 2 -times, changing any framing by \pm 2 , and introducing or erasing an unknot with \pm 2 framing.

The obvious question is that what kind of equivalence relation among 3-manifolds does $\mathbb{Z}_{2}$-equivalence of framed links induce? The following says that $\mathbb{Z}_{2}$-equivalence implies the birational equivalence.

Proposition 1. Any $\mathbb{Z}_{2}$-equivalent framed links represent birationally equivalent 3-manifolds.

Proof. Clearly introducing an unknot with \pm 2 framing corresponds to blowing up a point. Hence it suffices to show that twisting any strand of the framed link \pm 2 -times preserves birational equivalence. We must show that the manifolds $M_{1}$, and $M_{2}$ of Fig. 17 are birationally equivalent. This can be seen by blowing up both $M_{i}$, 
$i=1,2$, along the loops $\gamma_{i}, i=1,2$, respectively, cancelling the resulting 2-handles, and twisting as indicated in Fig. 18

Corollary 2. Any unorientable 3-manifold is birationally equivalent to an orientable 3-manifold.

Proof. First of all by introducing a cancelling pair of 2-handles as in Fig. 14 and sliding components of the framed link over one of them ( $\alpha$ in the picture), we can assume that every component of the framed link which goes over the 1-handle goes exactly twice. It suffices to show that by blowing up and down operations we can modify the framed link so that no component of the link goes over the 1-handle. Because then Case 2 would imply that the manifold is obtained by blowing up an oriented manifold along an unknot in a chart. Suppose a component $\theta$ of the link goes over the 1-handle as in Fig. 19. The box in the figure represents the complicated part of the framed link. We first attach a cancelling pair of 2-handles as in Fig. 20. By swinging over one of the balls of the 1-handle, we can put the unknotted circle with 0 -framing inside the box. Hence we can simplify Fig. 20 as Fig. 21. By the obvious handle slide we get Fig. 22. By blowing up the orientation reversing core circle of the 1-handle we get Fig. 23 (recall Case 5). A handle slide gives Fig. 24. By Proposition 1, Fig. 25 is birationally equivalent to Fig. 24, and a blowing down operation turns Fig. 25 into Fig. 26 (again as Case 5). Hence we freed $\theta$ from the 1-handle without introducing new framed knots going through the handle, i.e. we reduced the number of components of the framed link going through the 1-handle. Repeated application of this procedure gives the result.

4. Nakanishi's theorem and conclusion. We first need the following definition:

Definition. Let $K=\left\{K_{1}, \ldots, K_{n}\right\}$ and, $L=\left\{L_{1}, \ldots, L_{n}\right\}$ be links in $S^{3}$. We say that $K$ and $L$ are 2-homologous if the linking numbers satisfy the relation $\operatorname{lk}\left(K_{i}, K_{j}\right)=\operatorname{lk}\left(L_{i}, L_{j}\right) \bmod (2)$, for every $1 \leq i<j \leq n$. We call $K$ and $L$ 2-equivalent if one is obtained from the other by a sequence of operations of twisting across any strand \pm 2 times.

The following theorem of Nakanishi [N] says that these two concepts are equivalent. 
THEOREM 3 (Nakanishi). Any two links of the same number of components in $S^{3}$ are 2-equivalent if and only if they are 2-homologous.

This theorem follows from the amazing observation of [N] that the two strands of Fig. 27 are 2-equivalent, as shown in Fig. 28. For example, right away this shows that any knot is 2-equivalent to the unknot. More generally, this trick with [MN] gives the result. link.

COROLlary 4. Any framed link in $S^{3}$ is $\mathbb{Z}_{2}$ equivalent to the empty

Proof. By Theorem 3 any framed link is 2-equivalent to a framed link $K$ which consists of unknotted components with 1 or 0 framing, and pairwise components of $K$ link each other geometrically 1 or 0 times. Then, clearly by blowing down 1 framed circles (in the sense of the Kirby calculus), surgering unkotted components with 0 framing, and using Theorem 3, we can reduce the framed link to a link $L$ which consists of a disjoint union of unknots with 0 framings. Clearly $L$ is $\mathbb{Z}_{2}$-equivalent to the empty link.

THEOREM 5. Any two closed 3-manifolds are birationally equivalent.

Proof. It suffices to show that any closed 3-manifold $M$ is birationally equivalent to $S^{3}$. By Corollary 2 it suffices to assume that $M$ is orientable, so $M$ can be represented by a framed link in $S^{3}$, then Corollary 4 with Proposition 1 gives the result.

We would like to conclude this paper with the following remark: If $\varphi: U \rightarrow \mathbb{R}^{3}$ is a chart, with coordinates $\varphi=\left(\varphi_{1}, \varphi_{2}, \varphi_{3}\right)$, then blowing up $M$ produces new charts from $U$ whose transition functions are monomials in $\varphi_{i}, i=1,2,3$ with integer coefficients (they can be negative). The Nash conjecture says that $M$ is birationally equivalent to $S^{3}$, so this means that by extending the transition functions of the charts of $M$ by monomials we can make them equivalent to monomials. The implication of this in the context of geometric structures on 3-manifolds is yet to be understood.

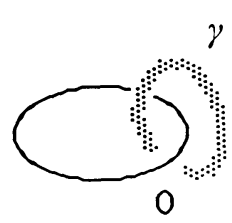

FIGURE 4

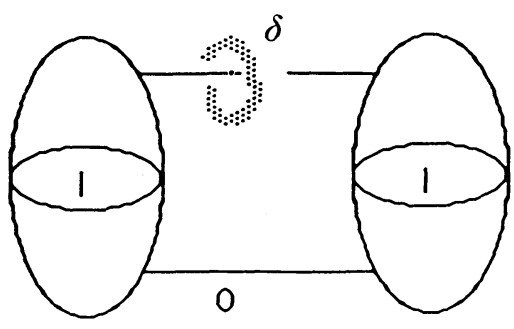

FIGURE 5 


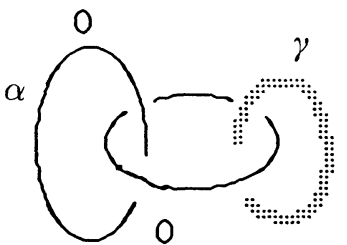

FIGURE 6

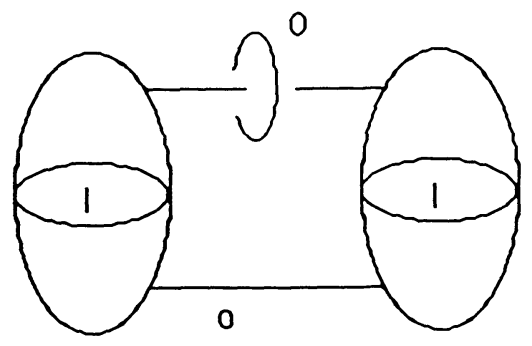

FIGURE 7
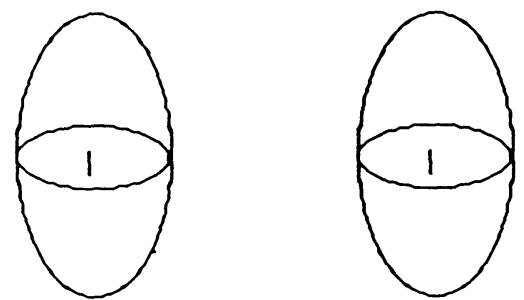

Figure 8

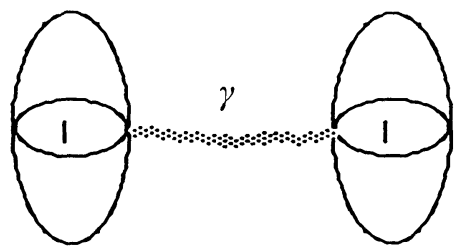

Figure 9

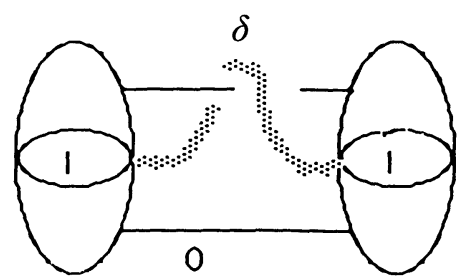

FIGURE 10

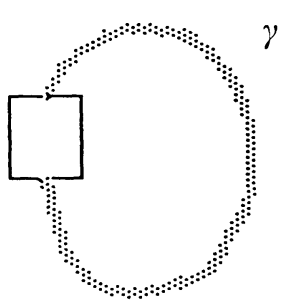

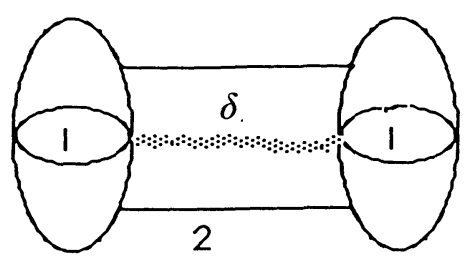

FIGURE 11

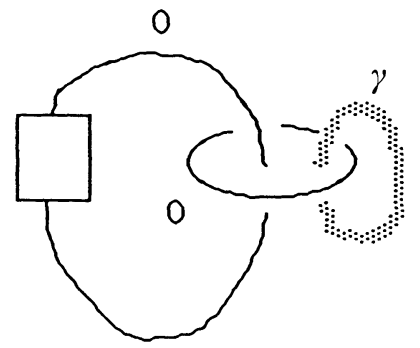

FIGURE 12 


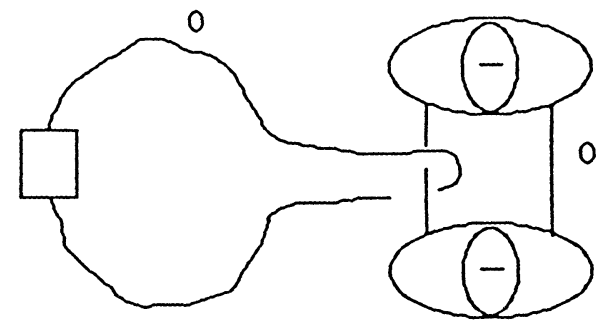

FIGURE 13

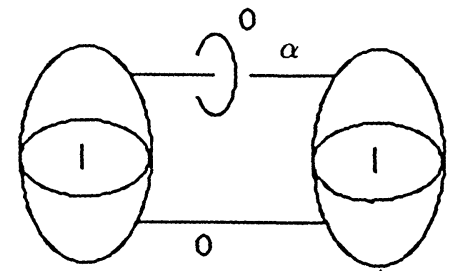

Figure 14

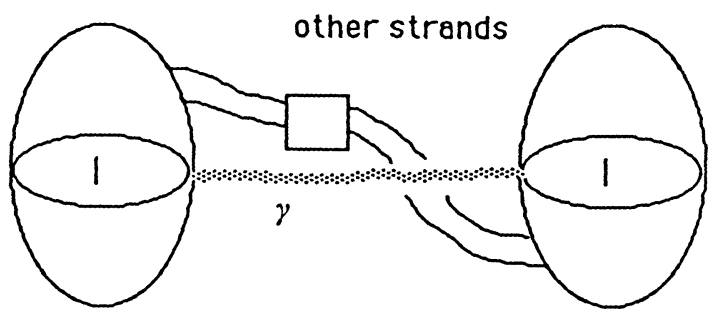

Figure 15

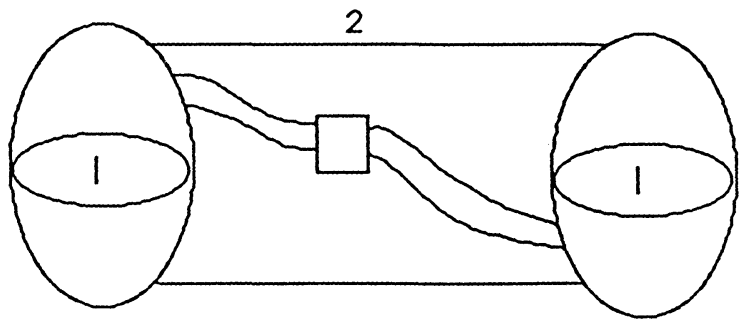

FIGURE 16

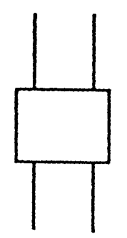

$M_{1}$

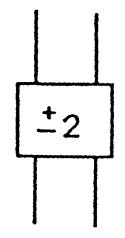

$M_{2}$

FIGURE 17 
210

SELMAN AKBULUT AND HENRY KING
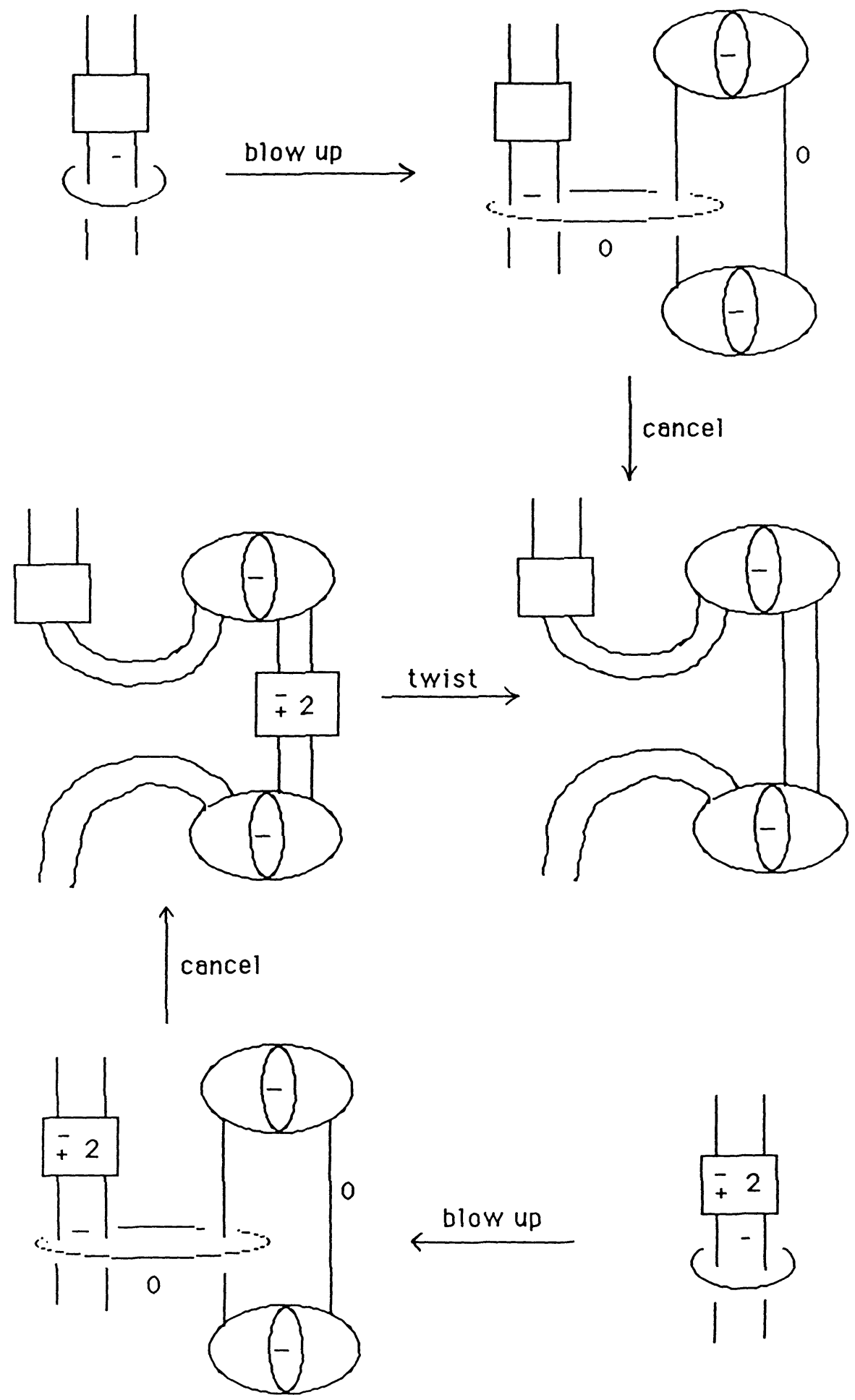

FIGURE 18 


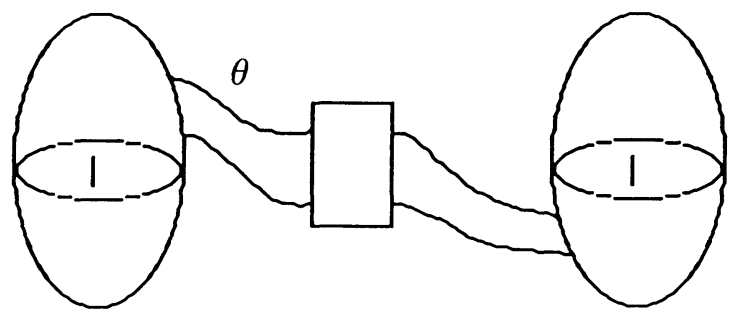

FIGURE 19

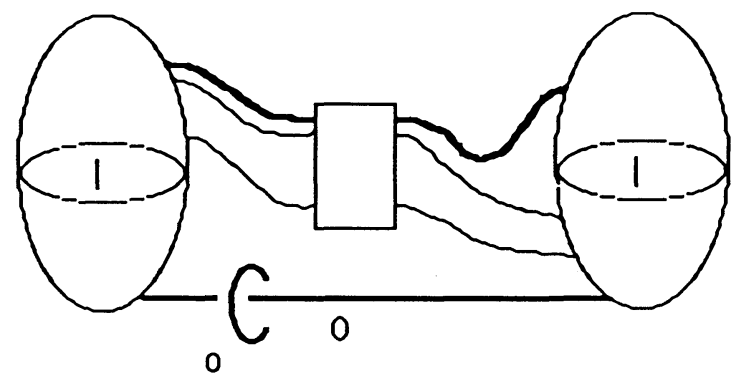

FIGURE 20

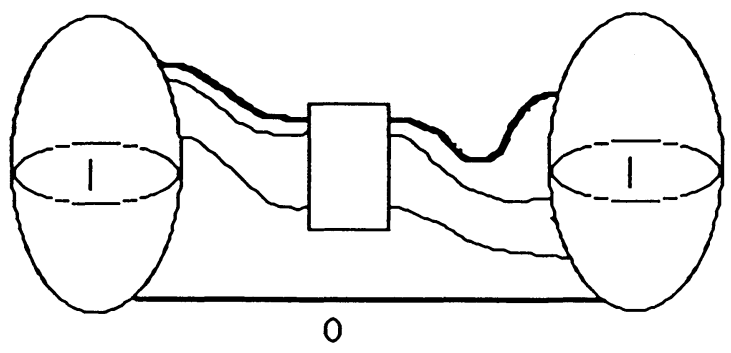

FigURE 21

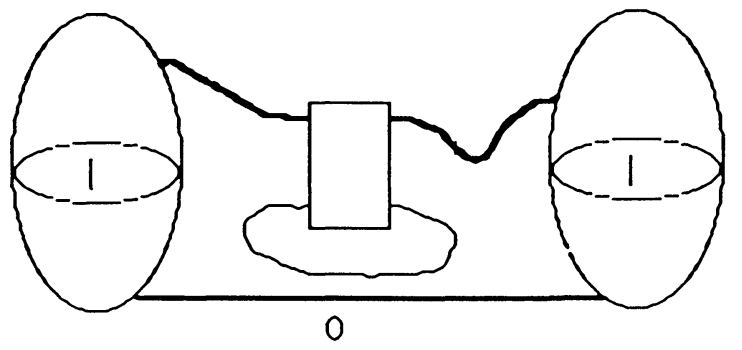

FIGURE 22 


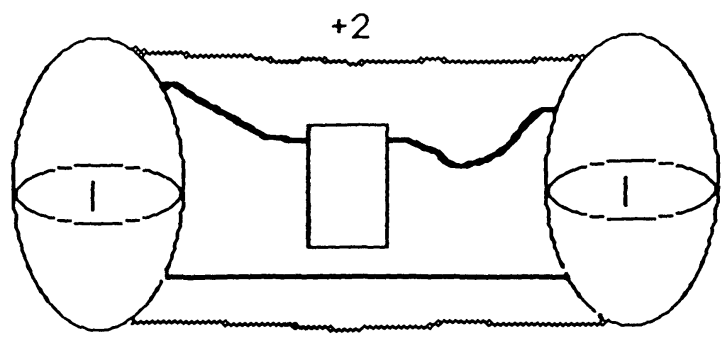

Figure 23

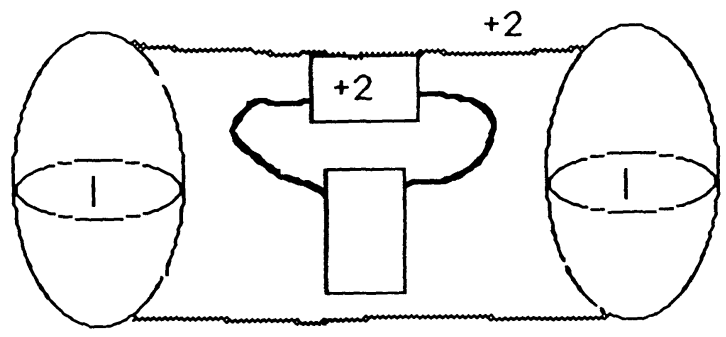

FIGURE 24

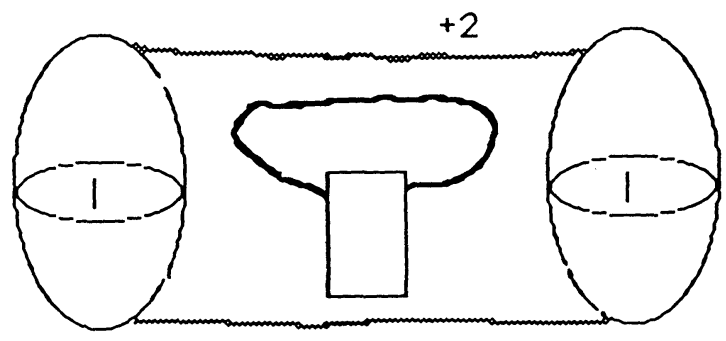

FIGURE 25

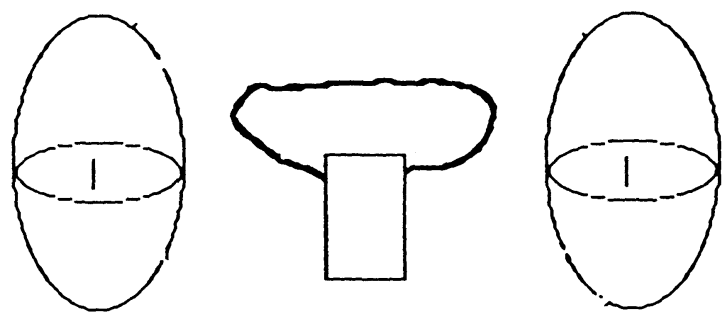

Figure 26
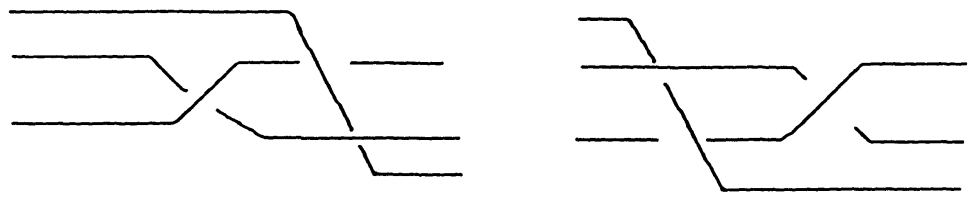

FigURE 27 


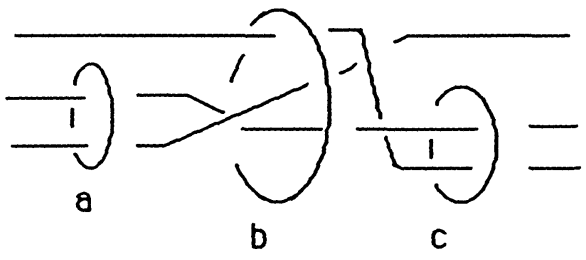

twist $a$ and $c+2$ times

twist $b-2$ times

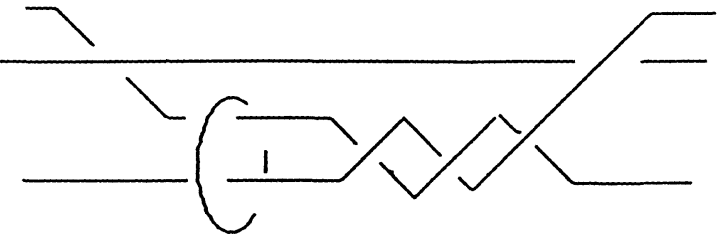

d

twist $d+2$ times

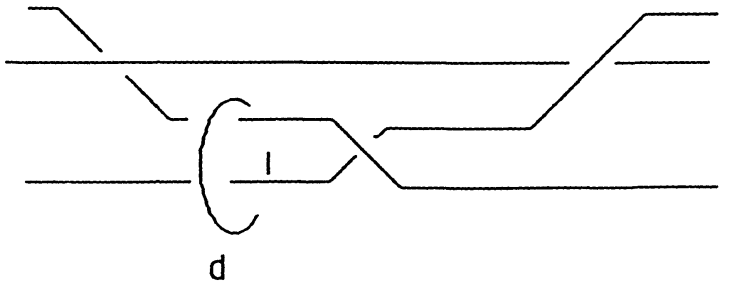

Figure 28

\section{REFERENCES}

[A1] S. Akbulut, $\mathbb{Z}_{2}$-framed link theory, Abstracts Amer. Math. Soc., (Feb. 1987), Issue 49, vol. 8, no. 2, p. 183.

[A2] _ A fake 4-manifold, Contemporary Math., 35 (1984), 75-141.

[K] R. Kirby, Calculus of framed links in $S^{3}$, Invent. Math., 45 (1978), 35-56.

[MN] H. Murakami and Y. Nakanishi, A certain move generating link-homology, (to appear Math. Ann.). 
[N] Y. Nakanishi, On Fox's congruence classes of knots 2, (to appear Osaka J. Math.).

[Na] J. Nash, Real algebraic manifolds, Ann. of Math., 56 (1952), 405-421.

[T] A. Tognoli, Some results on real algebraic cycles, Rocky Mountain J. Math., 14, no. 4, (Fall 1984), 833-843.

Received February 1, 1990 and in revised form April 30, 1990. Both authors are partially supported by NSF Grants.

Michigan State University

EAST LANSING, MI 48824

AND

UNIVERSITY OF MARYLAND

COllege PARK, MD 20742 


\section{PACIFIC JOURNAL OF MATHEMATICS EDITORS}

\author{
V. S. VARADARAJAN \\ (Managing Editor) \\ University of California \\ Los Angeles, CA 90024-1555-05 \\ Herbert Clemens \\ University of Utah \\ Salt Lake City, UT 84112 \\ THOMAS ENRIGHT \\ University of California, San Diego \\ La Jolla, CA 92093
}

R. FINN

Stanford University

Stanford, CA 94305

HeRmann FlaschKa

University of Arizona

Tucson, AZ 85721

VAUGHAN F. R. JoNES

University of California

Berkeley, CA 94720

SteVen KeRCKHOFF

Stanford University

Stanford, CA 94305
C. C. MOORE

University of California

Berkeley, CA 94720

MaRTin ScharLemanN

University of California

Santa Barbara, CA 93106

HAROLd STARK

University of California, San Diego

La Jolla, CA 92093

\section{ASSOCIATE EDITORS \\ R. ARENS \\ E. F. BECKENBACH (1906-1982) \\ B. H. NeumanN \\ F. WoLf \\ (1904-1989) \\ K. YoshidA \\ SUPPORTING INSTITUTIONS \\ UNIVERSITY OF ARIZONA \\ UNIVERSITY OF BRITISH COLUMBIA \\ CALIFORNIA INSTITUTE OF TECHNOLOGY \\ UNIVERSITY OF CALIFORNIA \\ MONTANA STATE UNIVERSITY \\ UNIVERSITY OF NEVADA, RENO \\ NEW MEXICO STATE UNIVERSITY OREGON STATE UNIVERSITY \\ UNIVERSITY OF OREGON \\ UNIVERSITY OF SOUTHERN CALIFORNIA \\ STANFORD UNIVERSITY \\ UNIVERSITY OF HAWAII \\ UNIVERSITY OF TOKYO \\ UNIVERSITY OF UTAH \\ WASHINGTON STATE UNIVERSITY UNIVERSITY OF WASHINGTON}

The Supporting Institutions listed above contribute to the cost of publication of this Journal, but they are not owners or publishers and have no responsibility for its content or policies.

Mathematical papers intended for publication in the Pacific Journal of Mathematics should be in typed form or offset-reproduced (not dittoed), double spaced with large margins. Please do not use built up fractions in the text of the manuscript. However, you may use them in the displayed equations. Underline Greek letters in red, German in green, and script in blue. The first paragraph must be capable of being used separately as a synopsis of the entire paper. In particular it should contain no bibliographic references. Please propose a heading for the odd numbered pages of less than 35 characters. Manuscripts, in triplicate, may be sent to any one of the editors. Please classify according to the 1991 Mathematics Subject Classification scheme which can be found in the December index volumes of Mathematical Reviews. Supply name and address of author to whom proofs should be sent. All other communications should be addressed to the managing editor, or Elaine Barth, University of California, Los Angeles, California 90024-1555-05.

There are page-charges associated with articles appearing in the Pacific Journal of Mathematics. These charges are expected to be paid by the author's University, Government Agency or Company. If the author or authors do not have access to such Institutional support these charges are waived. Single authors will receive 50 free reprints; joint authors will receive a total of 100 free reprints. Additional copies may be obtained at cost in multiples of 50 .

The Pacific Journal of Mathematics (ISSN 0030-8730) is published monthly except for July and August. Regular subscription rate: $\$ 190.00$ a year (10 issues). Special rate: $\$ 95.00$ a year to individual members of supporting institutions.

Subscriptions, orders for numbers issued in the last three calendar years, and changes of address should be sent to Pacific Journal of Mathematics, P.O. Box 969, Carmel Valley, CA 93924, U.S.A. Old back numbers obtainable from Kraus Periodicals Co., Route 100, Millwood, NY 10546.

The Pacific Journal of Mathematics at P.O. Box 969, Carmel Valley, CA 93924 (ISSN 0030-8730) is published monthly except for July and August. Second-class postage paid at Carmel Valley, California 93924, and additional mailing offices. Postmaster: send address changes to Pacific Journal of Mathematics, P.O. Box 969, Carmel Valley, CA 93924.

PUBLISHED BY PACIFIC JOURNAL OF MATHEMATICS, A NON-PROFIT CORPORATION Copyright (C) 1991 by Pacific Journal of Mathematics 


\section{Pacific Journal of Mathematics}

Vol. 150, No. $2 \quad$ October, 1991

Selman Akbulut and Henry Churchill King, Rational structures on

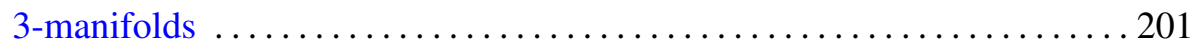

Mark Baker, On coverings of figure eight knot surgeries $\ldots \ldots \ldots \ldots \ldots 215$

Christopher Michael Brislawn, Traceable integral kernels on countably generated measure spaces . ...........................229

William Chin, Crossed products and generalized inner actions of Hopf algebras

Tadeusz Figiel, William Buhmann Johnson and Gideon Schechtman, Factorizations of natural embeddings of $l_{p}^{n}$ into $L_{r}$. II $\ldots \ldots \ldots \ldots \ldots 261$

David Howard Gluck, Character value estimates for groups of Lie type ... 279

Charn-Huen Kan, Norming vectors of linear operators between $L_{p}$

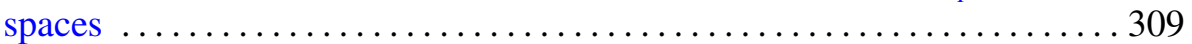

Marko Kranjc, Embedding a 2-complex $K$ in $\mathbb{R}^{4}$ when $H^{2}(K)$ is a cyclic

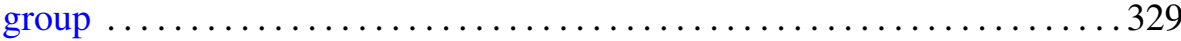

Ka-Lam Kueh, The remainder terms aspect of the theory of the Riemann

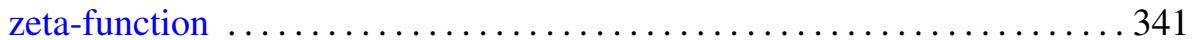

J. A. Marti, Sur la rigidité comparée de fonctions, distributions, ou hyperfonctions analytiques par rapport à un groupe de variables

Margherita Roggero and Paolo Valabrega, Chern classes and cohomology for rank 2 reflexive sheaves on $\mathbf{P}^{3}$ 